\title{
The PAS/LOV protein VIVID controls temperature compensation of circadian clock phase and development in Neurospora crassa
}

\author{
Suzanne M. Hunt, Mark Elvin, Susan K. Crosthwaite, and Christian Heintzen ${ }^{1}$ \\ Faculty of Life Sciences, The University of Manchester, Manchester M13 9PT, United Kingdom
}

\begin{abstract}
Circadian clocks are cellular timekeepers that regulate aspects of temporal organization on daily and seasonal time scales. To allow accurate time measurement, the period lengths of clocks are conserved in a range of temperatures-a phenomenon known as temperature compensation. Temperature compensation of circadian clock period aids in maintaining a stable "target time" or phase of clock-controlled events. Here we show that the Neurospora protein VIVID (VVD) buffers the circadian system against temperature fluctuations. In $v v d$-null mutants, the circadian period of clock-controlled events such as asexual sporulation (conidiation) is temperature compensated, but the phase of this clock time marker is not. Consistent with delayed conidiation at lower temperatures in $v v d^{\mathrm{KO}}$ strains, the levels of $v v d$ gene products in the wild type increase with decreasing temperatures. Moreover, $v v d^{\mathrm{C} 108 \mathrm{~A}}$ mutants that lack the light function of VVD maintain a dark activity that transiently influences the phase of conidiation, indicating that VVD influences the time of conidiation downstream from the clock. FREQUENCY (FRQ) phosphorylation is altered in a $v v d^{\mathrm{KO}}$ strain, suggesting a mechanism by which VVD can influence the timing of clock-controlled processes in the dark. Thus, temperature compensation of clock-controlled output is a key factor in maintaining temperature compensation of the entire circadian system.
\end{abstract}

[Keywords: Neurospora; circadian clock; temperature compensation; period; phase; VVD]

Supplemental material is available at http://www.genesdev.org.

Received April 16, 2007; revised version accepted June 22, 2007.

The survival of organisms that experience a wide range of daily and seasonal temperatures depends on a variety of strategies, including the prediction of and preparation for temperature change, maintenance of body temperature, and the temperature compensation of key biochemical and physiological processes (for review, see Rensing and Ruoff 2002; Somero 2004). Whereas many physical or physicochemical processes may be only slightly affected by changes in ambient temperature, chemical or biochemical reactions characteristically have temperature coefficients of $>2$; i.e., their reaction rates double with every $10^{\circ} \mathrm{C}$ rise in temperature (Snyder 1908). However, notable exceptions to this have been reported. For instance, aquatic poikilotherms frequently display metabolic temperature compensation (Newell 1966). At the level of enzyme activity, immediate compensation to temperature changes can be achieved by an

${ }^{1}$ Corresponding author.

E-MAIL christian.heintzen@manchester.ac.uk; FAX 44-0161-275-5082. Article is online at http://www.genesdev.org/cgi/doi/10.1101/gad.437107. inverse relationship between temperature and enzyme substrate affinity (Somero 1969). A striking example of temperature compensation at the behavioral level includes the escape response of the marine copepod Calanus finmarchius, which inhabits the high-latitude waters and experiences water temperatures from $<0^{\circ} \mathrm{C}$ to $18^{\circ} \mathrm{C}$. Whereas many aspects of this organism's biology have a $\mathrm{Q}_{10}$ of $>2$, the strongly developed escape response shows a much lower temperature dependence, presumably as an adaptation to cold-compensated predators (Lenz et al. 2005).

Other well-documented examples of temperature compensation relate to the periodicity of circadian clocks and the rhythms they control (Pittendrigh 1993). Temperature compensation of period, essential for an accurate timekeeper, is found in both homeothermic (Izumo et al. 2003; Tsuchiya et al. 2003) and poikilothermic organisms (Dunlap et al. 2004; Onai et al. 2004; Heintzen and Liu 2007).

In addition to light, temperature is one of the main environmental cues that synchronize circadian clocks to the rhythmic environment (Dunlap et al. 2004). This 
synchronization, or entrainment, is due to the coupling of the endogenous clock to the rhythmic light-dark (LD) and temperature changes of the external day, such that a stable phase relationship to the cyclical environment is established (Johnson et al. 2003; Roenneberg et al. 2003). Entrainment is an important characteristic of circadian clocks, as it ensures that clock-controlled processes occur at appropriate times of day. For many organisms in which circadian clocks are studied at the molecular level, the sensors and modulators of light signaling pathways that are critical for clock resetting and entrainment are known (Reppert and Weaver 2001; Young and Kay 2001; Golden 2003; Dunlap and Loros 2004; Liu and BellPedersen 2006). In contrast, our knowledge of components that mediate temperature control of circadian systems is still relatively sparse, despite recent studies that have concentrated on the effects of temperature on the circadian clockwork (Bell-Pedersen et al. 2005; Brunner and Diernfellner 2006; Hotta et al. 2007).

In addition to acting as an entrainment cue, temperature sets limits for clock function beyond which rhythmicity is no longer supported. Moreover, temperature must influence the processes that contribute to the phenomenon of temperature compensation-a poorly understood mechanism that allows circadian clocks to maintain a relatively stable periodicity over a wide range of ambient temperatures (Dunlap and Loros 2004). One way of addressing the problem of temperature compensation is to model the known effects of temperature on clock molecules. This approach has been taken by several laboratories (Ruoff et al. 2005a,b; Gould et al. 2006). In Arabidopsis, quantitative data on the temperaturedependent expression of clock mRNAs are consistent with the antagonistic balance model of temperature compensation (Ruoff 1992; Gould et al. 2006). In Neurospora, the stability of the key clock protein FREQUENCY (FRQ) plays a pivotal role in temperature compensation (Ruoff et al. 2005b). However, although these studies have given us some insight into the possible mechanisms of temperature compensation, only a subset of clock molecules have been incorporated into these models.

In Neurospora, the products of the frequency (frq) locus have been implicated in mediating all three temperature effects on the Neurospora circadian clock (Liu et al. 1997; Kramer et al. 2003; Colot et al. 2005; Diernfellner et al. 2005). The frq gene encodes for two forms of FRQ protein, a large 989-amino-acid FRQ form (lFRQ) and a small FRQ form (sFRQ) that lacks the first 100 amino acids of full-length FRQ. The amount of 1FRQ is regulated by temperature. Temperature-regulated splicing of an intron deletes the ATG for 1FRQ and leads to a relative increase in the ratio of $1 F R Q: s F R Q$ with increasing temperature (Colot et al. 2005; Diernfellner et al. 2005). Additionally, temperature-dependent translational control mediated by upstream ORFs (uORFs) in the frq $5^{\prime}$ untranslated region (UTR) leads to a further increase of lFRQ levels with rising temperature (Diernfellner et al. 2005). At a high temperature, these nonconsensus uORFs are less efficiently translated, and consequently there is an increase in translation efficiency of FRQ. The initial promise of these data in explaining aspects of temperature compensation, however, has not been realized, as either sFRQ or IFRQ alone allow the Neurospora clock to be temperature compensated if present in sufficient quantities (Liu et al. 1997; Colot et al. 2005; Diernfellner et al. 2005).

Interestingly, some clock-controlled and temperatureentrainable genes have lost their temperature responsiveness in frq-null strains, an observation that is consistent with (but not proof of) a role of FRQ as a temperature sensor (Nowrousian et al. 2003). To summarize, although FRQ appears to play a key role in mediating temperature responses of the circadian clock, we know little about the signaling events that transduce changes in temperature to alter FRQ function. Moreover, frq-independent temperature-sensitive pathways may exist that help to entrain the Neurospora circadian system. It is known, for instance, that temperature changes can drive rhythmic conidiation in strains that lack a functional frq gene (Merrow et al. 1999; Pregueiro et al. 2005; Lakin-Thomas 2006). In addition, de Paula et al. (2006) have identified a gene named $c c g$-16 that undergoes temperature-compensated oscillations with circadian periodicity in the absence of frq.

In a temperature entrainment cycle, a common feature of circadian systems is that the relatively higher temperature is interpreted as daylight, whereas the relatively lower temperature mimics darkness. This similarity of light and temperature responses suggests that both cues may act to regulate the same entrainment pathways. For instance, a step up in temperature transiently induces frq RNA levels, surprisingly similar to the effects of light on frq RNA accumulation (Crosthwaite et al. 1995; Liu et al. 1998).

The PAS/LOV protein VIVID (VVD) was previously reported to influence the transient nature of the Neurospora light response, with consequences for resetting and entrainment of the circadian clock (Heintzen et al. 2001; Schwerdtfeger and Linden 2001; Shrode et al. 2001; Elvin et al. 2005). Here we show that vvd transcript and VVD protein levels are temperature regulated and influence the phosphorylation state of FRQ at the LD boundary, and that normal VVD expression ensures that the phase of the Neurospora clock remains stable in a wide range of physiological temperatures. In strains that lack VVD, the phosphorylation kinetics of FRQ are changed and the time of clock-controlled conidiation is no longer temperature compensated. Finally, the phenotype of a light signaling-defective strain, in which a cysteine required for VVD's photoactivation is mutated to an alanine $\left(v v d^{\mathrm{C} 108 \mathrm{~A}}\right)$, indicates that VVD has light- and dark-specific roles. Immediately after light-to-dark transfer, the phase of this strain is similar to the phase of the wild type; however, in continued darkness it establishes a constant but delayed phase to that of wild type. Thus, not only does VVD have light- and dark-specific roles that control the phase of the Neurospora clock at both dawn and dusk transitions, it also acts on temperature regulation of clock-controlled output pathways to ensure 
Hunt et al.

appropriate timing of clock-controlled development over a range of temperatures.

\section{Results}

VVD contributes to temperature compensation of circadian clock phase

The small PAS/LOV protein VVD has two functions: one as a photoreceptor and the other as a repressor of WHITE COLLAR-mediated light responses (Heintzen et al. 2001; Schwerdtfeger and Linden 2001, 2003; Shrode et al. 2001; Elvin et al. 2005; Zoltowski et al. 2007). As a modulator of light responses, VVD plays an important role during entrainment to LD cycles. At dawn, VVD mutes or prevents clock resetting, allowing the circadian clock to run through the LD transition and measure day length. At dusk, VVD promotes resetting by influencing the decay kinetics of frq RNA (Elvin et al. 2005).

Because light and high temperature reinforce each other as entrainment cues, with high temperature mimicking light and low temperature mimicking darkness, we wondered whether vvd mutant strains are also more sensitive to temperature pulses compared with wild-type strains. We exposed race tube cultures kept at $25^{\circ} \mathrm{C}$ in constant darkness (DD) to temperature step-up $\left(30^{\circ} \mathrm{C}\right)$ or temperature step-down $\left(20^{\circ} \mathrm{C}\right)$ pulses of 2 -h duration every $2 \mathrm{~h}$ during the first day of darkness. The effects of these temperature pulses were compared with those of unpulsed control cultures, and the data were plotted as a phase response curve (PRC). The results from these phase-shift experiments (Fig. 1A) are largely in line with early work by Francis and Sargent (1979), with step-up pulses resulting in delays of the clock toward the end of subjective day/early subjective night and advances during the subjective night (Francis and Sargent 1979). Although the phase shifts resulting from step-down pulses are fairly weak, they are similar to the phase shifts reported in the literature in that they seem to be shifted in time by $\sim 8-12 \mathrm{~h}$ when compared with the temperature pulse-up PRC. Our PRCs are generally weaker and more symmetrical than those obtained by Francis and Sargent (1979), which is not surprising considering that we have given far shorter temperature pulses ( $2 \mathrm{~h}$ vs. $6 \mathrm{~h}$ ). Importantly, we see no significant differences between the PRCs of both strains, suggesting that acute effects of temperature are not influenced by VVD.

To complete our investigation into the effect of temperature on $v v d$ mutants, we assayed temperature compensation by determining the free-running period (FRP) of clock wild-type and $v v d^{\mathrm{KO}}$ strains as well as $f r q^{7}$ (29h-long-period mutant) and $f r q^{7} ; v v d^{\mathrm{KO}}$ double mutants in temperatures of $16^{\circ} \mathrm{C}, 18^{\circ} \mathrm{C}, 21^{\circ} \mathrm{C}, 25^{\circ} \mathrm{C}, 28^{\circ} \mathrm{C}, 30^{\circ} \mathrm{C}$, or $32^{\circ} \mathrm{C}$ (Fig. 1B). Clock wild-type strains are temperature compensated under these conditions; i.e., they have a similar period length at all temperatures. In contrast, strains with defects in the central clockwork (such as the long-period mutant $f r q^{7}$ ) often show defects in temperature compensation (Gardner and Feldman 1981; Feldman et al. 1986). As expected, the $f r q^{7}$ strain and $f_{r} q^{7} ; v v d^{\mathrm{KO}}$
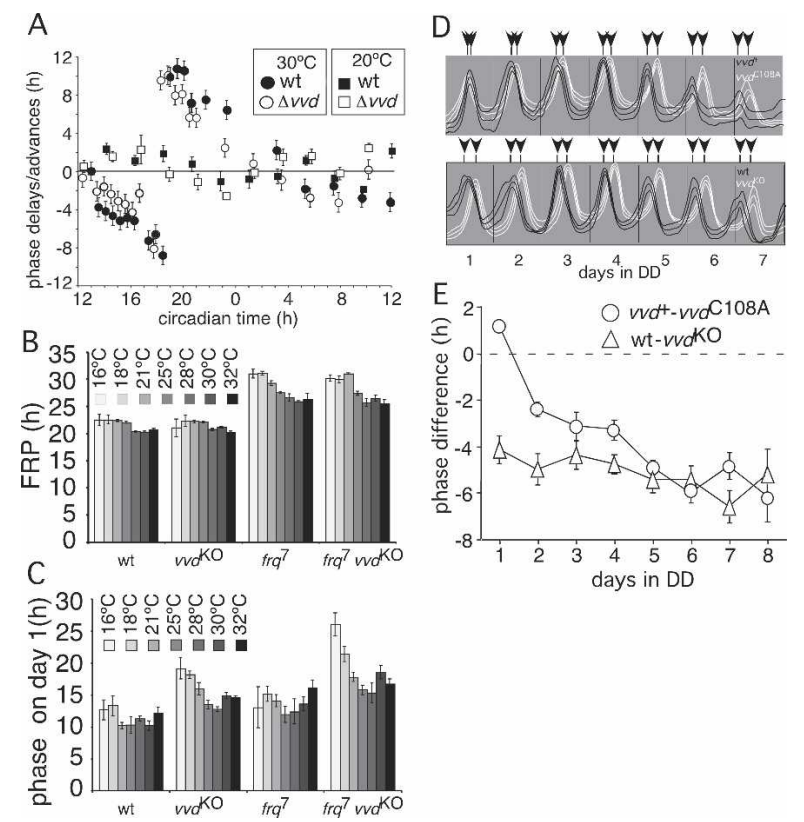

Figure 1. VVD affects temperature compensation of clock phase at the output level. (A) The response to temperature pulses is similar in wild type and a $v v d^{\mathrm{KO}}$ strain. PRCs were generated with 2 -h temperature pulse-up $\left(25^{\circ} \mathrm{C}-30^{\circ} \mathrm{C}\right)$ or pulsedown $\left(25^{\circ} \mathrm{C}-20^{\circ} \mathrm{C}\right)$ treatments as described in Materials and Methods. The PRC is plotted in CT with the center of the conidial band defined as CT0; i.e., subjective dawn. The PRC graphs the difference in circadian timing of conidiation (the position of the conidial band) in pulsed versus unpulsed controls. The abscissa shows CT in hours when temperature pulse was given, and the ordinate shows phase shift in hours of advance or delay. (Filled symbols) Wild type (wt); (open symbols) $v v d^{\mathrm{KO}}$; (rectangles) $20^{\circ} \mathrm{C}$; (circles) $30^{\circ} \mathrm{C}$ temperature pulse; (error bars) $\pm S D ; n=6-18$. $(B, C) V v d^{\mathrm{KO}}$ strains show defects in temperature compensation of clock phase but are temperature compensated for the period. Graph showing average period length $(B)$ and average phase on day one $(C)$ for wild-type $(w t), v v d^{\mathrm{KO}}, f r q^{7}$, and $f r q^{7}, v v d^{\mathrm{KO}}$ strains. $f r q^{7}$ is a long-period (29-h) mutant that has a known defect in temperature compensation of period. Note the increasingly delayed phase at the lower end of the temperature range in $v v d^{\mathrm{KO}}$ and $f r q^{7}, v v d^{\mathrm{KO}}$ strains. Error bars indicate $\pm 1 \mathrm{SD}$. $(D, E)$ VVD affects time of conidiation at the output level. $(D$, top panel) Densitometric traces of race tube cultures of $v v d^{+}$(black traces) and $v v d^{\mathrm{C} 108 \mathrm{~A}}$ (white traces) strains. Arrows above the traces highlight the peaks of conidiation. Notice the identical phase on day 1 of both strains, but subsequent establishment of a constant phase delay between strains. (Bottom panel) As above, but race tube cultures are those of $v v d^{+}$(black traces) and $v v d^{\mathrm{KO}}$ (white traces) strains. Notice the constant phase delay of $v v d^{\mathrm{KO}}$ strains compared with wild type. (Center trace) Mean; (outside traces) \pm 1 SE; $n=6$. $(E)$ Graph showing the differences in peaks of conidiation (in hours) between $v v d^{+}$and $v v d$ mutant strains plotted against the number of days in DD. Error bars indicate \pm 1 SD. (Broken line) Line of identical phase.

show defects in temperature compensation. Similar to the wild type, the $v v d^{\mathrm{KO}}$ is temperature compensated; however, when looking at the phase (time of conidiation peak in the first day in DD), we noticed a strong depen- 
dency on temperature in both the $v v d^{\mathrm{KO}}$ and $f r q^{7} ; v v d^{\mathrm{KO}}$ double mutants (Fig. 1C). Conidiation becomes noticeably delayed at the lower end of the temperature range, and temperature dependence is particularly striking in $f r q^{7} ; v v d^{\mathrm{KO}}$ double mutants.

We wondered whether part of VVD's role was to compensate for such temperature-dependent effects by affecting conidiation downstream from the clock. To study this, we generated a strain that expressed a mutant VVD protein in which a cysteine critical for photosignaling, and thus for VVD's light functions, was mutated to an alanine. A similar mutant was previously shown to mimic the light-dependent phenotypes of a $v V d$ knockout strain-e.g., increased carotenogenesis and defects in photoadaptation-but was not investigated for any clock phenotypes (Schwerdtfeger and Linden 2003). We expected that light-mediated clock resetting would be similar in $v v d^{\mathrm{C} 108 \mathrm{~A}}$ and $v v d^{\mathrm{KO}}$ strains-i.e., delayed by $\sim 4 \mathrm{~h}$-because the light-specific functions of VVD are abolished. On the other hand, since the VVD ${ }^{\mathrm{C} 108 \mathrm{~A}}$ may be functional in the dark we wondered whether, upon release to DD, the mutant VVD could mask true clock phase by influencing conidiation at the output level. As we have shown previously and in this study that $v v d$ transcript and VVD protein are only detectable during the first day in darkness, we would expect this influence of VVD on the timing of conidiation to be transient (Heintzen et al. 2001; Elvin et al. 2005). As shown in Figure 1, D and E, the phase of conidiation of the $V V d^{\mathrm{C} 108 \mathrm{~A}}$ strain in the first day in DD is similar to wild type and different from that of a $v v d^{\mathrm{KO}}$ strain. However, this difference is transient, and after $4 \mathrm{~d}$ in DD the $V v d^{\text {C108A }}$ has established a $4-h$ phase delay and conidiates with a similar phase as the $v v d^{\mathrm{KO}}$ strain. The data shown in Supplementary Figure S1 supports the above interpretation. In this experiment, the timing of conidiation of wild type and $v v d^{\mathrm{KO}}$ grown in the light at $18^{\circ} \mathrm{C}$ or $30^{\circ} \mathrm{C}$ and then transferred to $\mathrm{DD} 30^{\circ} \mathrm{C}$ was compared. The timing of conidiation in the $v v d^{\mathrm{KO}}$ strain in the first day in DD occurs later than in the cultures transferred from $\mathrm{LL} 18^{\circ} \mathrm{C}$ compared with cultures transferred from LL $30^{\circ} \mathrm{C}$. However, in subsequent days the peaks of conidiation move together, reflecting the true time to which the clock was set at the LD transition. This again demonstrates that, in the wild-type, VVD acts downstream from the clock to influence the timing of conidiation and that VVD helps to counteract the effects of temperature on this developmental pathway. These results have a number of implications: First, photoactive VVD is required for normal resetting at the LD boundary; second, VVD has light- and dark-specific roles that can be separated; and third, VVD helps to preserve clock phase in different temperatures by influencing the phase of conidiation (at least in part) at the output level.

\section{vvd gene products are temperature regulated}

The defect in temperature compensation of phase in $v v d$ null strains prompted us to investigate whether the products of the $v v d$ gene themselves are temperature regu- lated. We grew Neurospora in liquid cultures for up to 36 $\mathrm{h}$ in constant light (LL) at $16^{\circ} \mathrm{C}, 21^{\circ} \mathrm{C}, 25^{\circ} \mathrm{C}$, or $28^{\circ} \mathrm{C}$ before turning the lights off and harvesting tissue at 4-h intervals in DD over a period of $24 \mathrm{~h}$. The Northern blots probed for $v v d$ transcript and their quantitative analyses are shown in Figure 2. It is clear that $v v d$ RNA levels are higher at lower temperatures. As previously reported (Heintzen et al. 2001), vvd transcript levels peak at DD12 (Fig. 2A,B), and our temperature experiments now show that the amplitude of this oscillation is temperature dependent (Fig. 2C). Figure 2D highlights the differences in $v v d$ transcript levels at times of peak expression (DD12) in cultures incubated at $16^{\circ} \mathrm{C}, 21^{\circ} \mathrm{C}, 25^{\circ} \mathrm{C}$, or $28^{\circ} \mathrm{C}$. Figure $2 \mathrm{E}$ documents the dramatic increase $(\sim 15-$ fold) in $v v d$ transcript levels at $16^{\circ} \mathrm{C}$ when compared with $28^{\circ} \mathrm{C}$. Interestingly, the strongest incremental increase is seen between $25^{\circ} \mathrm{C}$ and $21^{\circ} \mathrm{C}$, which coincides well with the dramatic delay in the time (i.e., phase) of conidiation (see Fig. 1C) seen on race tubes in $v v d^{\mathrm{KO}}$ strains at precisely this boundary.

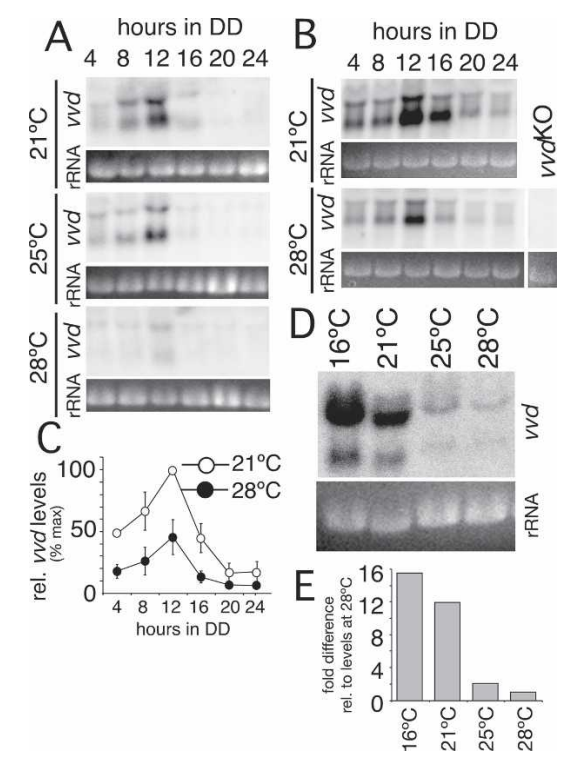

Figure 2. $\quad v v d$ transcript levels are temperature regulated. $(A)$ Northern blots showing $v V d$ transcript levels in the first day of $\mathrm{DD}$ at the indicated temperatures. Mycelial discs were grown in liquid culture at $21^{\circ} \mathrm{C}, 25^{\circ} \mathrm{C}$, and $28^{\circ} \mathrm{C}$, and tissue was harvested at the indicated time points in DD. Transcript levels peak around DD12 and decline below detection limits by DD24. Two transcripts can be distinguished. $(B)$ Experiment as described in $A$, but only $21^{\circ} \mathrm{C}$ and $28^{\circ} \mathrm{C}$ data are shown and blots are overexposed to highlight the difference at extreme temperatures. Total RNA from a $v v d^{\mathrm{KO}}$ strain was loaded to control for signal specificity. (C) Quantitative analysis of Northern blot data from three independent experiments graphed as percentage of maximal expression. Error bars indicate \pm 1 SD. $(D)$ Levels of $v v d$ transcript extracted from tissue harvested at DD12 at the indicated temperatures. (E) Quantitative analysis of Northern blot data shown in $D$. The lowest transcript levels (at $28^{\circ} \mathrm{C}$ ) are set to 1 to document the fold increase with decreasing temperature. All Northern blots are accompanied by panels of corresponding ethidium-bromide (EtBr)-stained ribosomal RNA (rRNA) to document loading. 
To complement our RNA expression studies, we wanted to know how VVD protein is regulated at various ambient temperatures in the dark. For this purpose, we generated strains in which a construct expressing a myctagged VVD protein was inserted at the his-3 locus of a $v v d^{\mathrm{KO}}$ strain. The construction of such a strain was necessary, as we and others have been unable to detect VVD protein in DD when using antibodies raised against bacterially expressed VVD protein (Heintzen et al. 2001; Schwerdtfeger and Linden 2003), although indirect evidence indicates that VVD must be expressed and function in the first day in DD (Elvin et al. 2005). MYCtagged proteins have been widely and successfully used in Neurospora to complement respective knockout strains (e.g., see Cheng et al. 2001, 2002). We generated a strain that expresses a MYC-tagged VVD protein in a $v v d^{\mathrm{KO}}$ background (see Materials and Methods). This

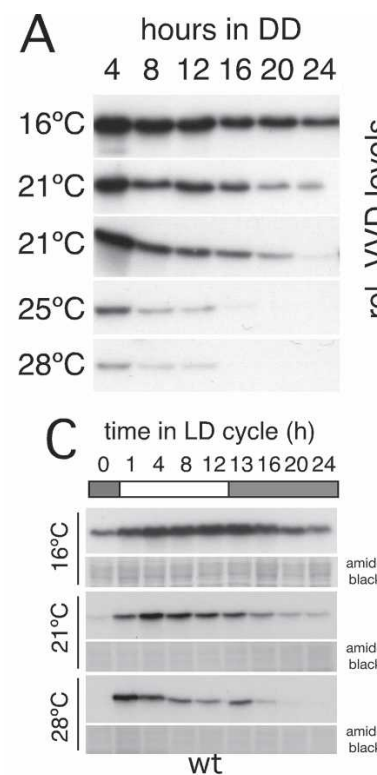

wt
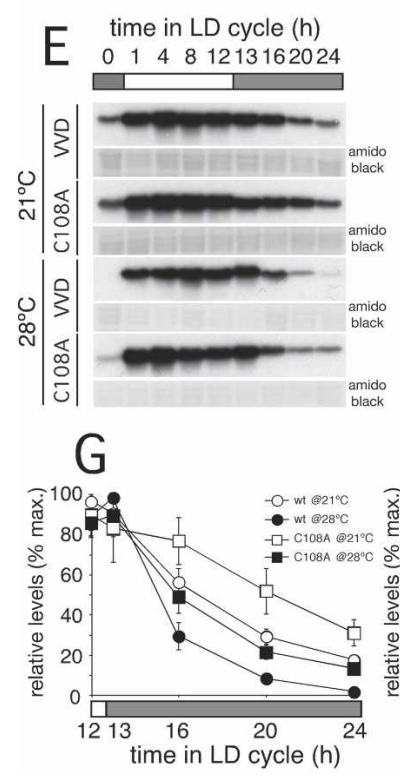

B
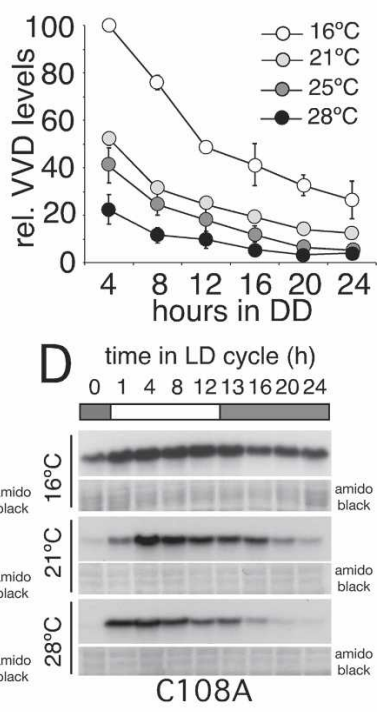

C108A

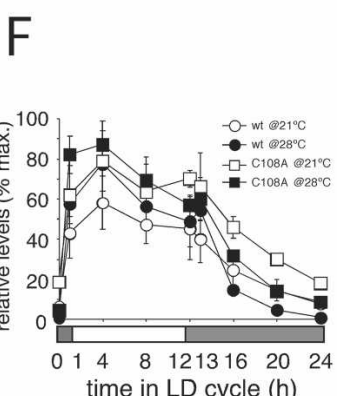

$\mathrm{H}$

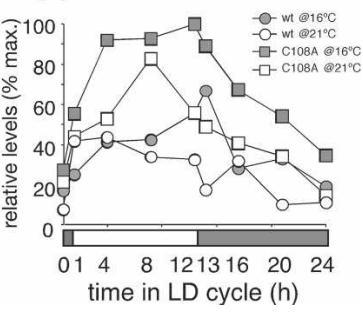

strain fully complements all known $v v d^{\mathrm{KO}}$ phenotypes, such as overaccumulation of colored carotenoids, delayed onset of conidiation, and hypersensitivity to light resetting (see Supplementary Fig. S2).

Similar to $v v d$ transcript levels, there is a dramatic increase in VVD protein levels at the lower end of the temperature range (Fig. 3A). However, whereas the vvd transcript shows a transient peak in expression at DD12, VVD protein decays steadily from the high levels seen immediately after the transfer of cultures from light to DD (Fig. 3A,B). VVD levels are significantly higher at $21^{\circ} \mathrm{C}$ at DD24 than at $28^{\circ} \mathrm{C}$ at DD24. For this time point, VVD levels at $21^{\circ} \mathrm{C}$ are roughly equivalent to levels reached at $28^{\circ} \mathrm{C}$ at $\mathrm{DD} 4$ (Fig. 3B). The differences become even greater when comparing $16^{\circ} \mathrm{C}$ with $28^{\circ} \mathrm{C}$. To test whether the higher levels of VVD seen at the lower temperatures are due to increased VVD levels accumulating in the preceding light and temperature conditions, we grew cultures in LL at $25^{\circ} \mathrm{C}$ before releasing them into $\mathrm{DD}$ at either $21^{\circ} \mathrm{C}, 25^{\circ} \mathrm{C}$, or $28^{\circ} \mathrm{C}$. Thus, VVD protein started from similar levels before experiencing the different temperatures in DD. As can be seen in Supplementary Figure S3, the degradation rate of VVD is temperature dependent; i.e., it degrades faster at higher temperatures. However, the overall increase in VVD protein is likely to be due to a combination of increased translation and a decreased degradation rate at the lower temperatures.

To test whether this temperature regulation of VVD is also seen in a LD environment, extracts were harvested at regular intervals from cultures grown in a 12-h light:12-h dark (12:12 LD) cycle (Fig. 3C). As expected VVD protein accumulates in the light (Heintzen et al. 2001; Elvin et al. 2005). The temperature-dependent deg-

Figure 3. VVD protein levels are temperature regulated. $(A)$ Western blot analysis following VVD levels in DD at the indicated temperatures. Cultures were grown as described in Figure 2A. Fifteen micrograms of protein were loaded per lane and Western blots were hybridized with a MYC-specific monoclonal antibody. $(B)$ Quantitative analysis of three independent experiments, as shown in $A$, graphed as percentage of maximal expression at $16^{\circ} \mathrm{C}$ at DD4. Error bars indicate $\pm 1 \mathrm{SD} ; n=3 ; n=2$ for $16^{\circ} \mathrm{C}$. (C) Western blots showing VVD levels in 12:12 LD entrainment conditions at $16^{\circ} \mathrm{C}, 21^{\circ} \mathrm{C}$, and $28^{\circ} \mathrm{C}$. (D) Similar experiment as shown in $C$ showing VVD ${ }^{\mathrm{C} 108 \mathrm{~A}}$ levels. (E) Similar experiment as in $C$ and $D$ with VVD wild-type (VVD) and VVD $^{\text {C108A }}$ (C108A) samples loaded on the same gel to visualize the differences in protein levels between both strains at $21^{\circ} \mathrm{C}$ and $28^{\circ} \mathrm{C}$. VVD ${ }^{\mathrm{C} 108 \mathrm{~A}}$ levels are higher than wild-type VVD levels at both temperatures. (F) Quantitative analysis of $21^{\circ} \mathrm{C}$ and $28^{\circ} \mathrm{C}$ data shown in $C-E ; n=3$ or 4 . Protein levels are normalized to maximum levels. (G) Same as in $F$, but protein levels are normalized to levels at LD12 (end of light period) to emphasize the kinetics of VVD turnover after the LD transition. $(H)$ Quantitative analysis of an experiment as described in $F$ performed at $16^{\circ} \mathrm{C}$ and $21^{\circ} \mathrm{C}$ and graphed as the percentage of maximal expression. VVD levels show a further increase when the temperature is lowered to $16^{\circ} \mathrm{C}$ and $\mathrm{VVD}^{\mathrm{C} 108 \mathrm{~A}}$ levels are again higher than VVD wild-type levels in all conditions. All error bars indicate \pm 1 SE. Black and white bars indicate lights off or on, respectively. 
radation of VVD in the dark is also evident in these entrainment conditions (Fig. 3G). Since VVD degrades more slowly at $16^{\circ} \mathrm{C}$ and $21^{\circ} \mathrm{C}$, there are still significant amounts of VVD detectable before dawn at these temperatures, whereas there is no detectable VVD protein at this time point at $28^{\circ} \mathrm{C}$ (Fig. $3 \mathrm{C}$ ).

Interestingly, the amount of VVD present has consequences for the kinetics of VVD induction in the light. At the relatively higher temperatures-i.e., when VVD is undetectable at the end of the night-light induction of VVD is faster, reaching a maximum relatively early (LL1-LL4) (see Fig. 3C-F). In contrast, at low temperatures and with significant amounts of VVD still present before dawn, light induction of VVD is much slower, with maximum levels at $16^{\circ} \mathrm{C}$ occurring at about LL8LL12 (e.g., see Fig. 3C, top panel). We also investigated the levels of the $\mathrm{VVD}^{\mathrm{C} 108 \mathrm{~A}}$ mutant protein in these conditions. Similar to the strategy mentioned above, a myctagged version of the mutated gene was expressed in a $v v d^{\mathrm{KO}}$ strain. As described previously, this strain does not rescue the carotenoid phenotype of the knockout strain (Schwerdtfeger and Linden 2003; data not shown). Levels of $\mathrm{VVD}^{\mathrm{C} 108 \mathrm{~A}}$ are significantly elevated when compared with wild-type VVD (Fig. 3C-E). This is most pronounced at the higher temperatures toward the end of the night period (e.g., see Fig. 3E, cf. bottom two panels). Taken together, these results support the hypothesis that VVD negatively autoregulates its own expression.

The higher expression levels of VVD ${ }^{\mathrm{C} 108 \mathrm{~A}}$ could explain the early phase of conidiation of the $v v d^{\mathrm{C} 108 \mathrm{~A}} \mathrm{mu}$ tant during the first day in DD (Fig. 1D,E). The fact that $V v d^{\mathrm{C} 108 \mathrm{~A}}$ mutants eventually establish a phase that is similar to that of $v v d$ knockout strains is consistent with the hypothesis that VVD, at least in part, regulates the onset of conidiation at the output level. In this strain, during the first day of $\mathrm{DD}$ only the expression of a darkactive VVD (not requiring the photoactive cysteine) is required to establish the phase of conidiation, which is similar to that of wild type. However, as mutant VVD levels drop so does its influence on conidiation, and the true clock phase that was established at the LD boundary now becomes evident (see Fig. 1D,E). This clock phase would be reminiscent of a $v v d^{\mathrm{KO}}$ strain.

\section{Temperature regulation of $\mathrm{vvd}$ is not dependent on a functional frq gene}

Since the products of the $f r q$ gene influence temperature responses of the circadian clock (Liu et al. 1997; Colot et al. 2005; Diernfellner et al. 2005) we wondered whether temperature regulation of $v v d$ is dependent on a functional frq locus. To investigate this, we looked at temperature regulation of $v v d$ transcript in wild-type and frq ${ }^{10}$ (frq-null) strains. We found that temperature regulation of $v v d$ transcript is normal in frq-null $\left(f r q^{10}\right)$ strains, indicating that the $f r q$ gene is not essential for temperature regulation of $v v d$ (Fig. $4 \mathrm{~A}, \mathrm{~B}$ ), and that frqindependent pathways must confer temperature regulation of the vvd gene.

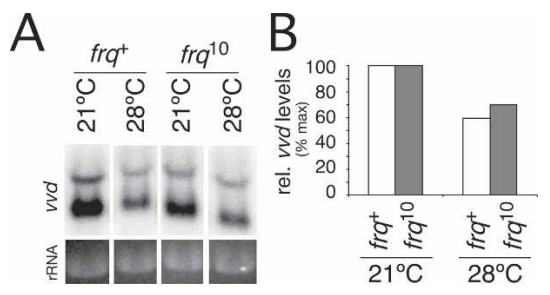

Figure 4. Temperature regulation of $v v d$ transcript levels is not dependent on a functional frq gene. $(A)$ Northern blot showing $v v d$ transcript levels in wild-type and $f_{r q}$-null $\left(f_{r} q^{10}\right)$ strains at DD12 (peak levels of $v v d$ ) at $21^{\circ} \mathrm{C}$ and $28^{\circ} \mathrm{C}$. EtBr-stained rRNA is shown to document even loading. (B) Quantitative analysis of Northern blot data shown in $A$. For each strain, vvd RNA levels at $21^{\circ} \mathrm{C}$ were set to $100 \%$.

vvd influences the dynamics of FRQ phosphorylation but not the ratio of FRQ forms

Because the clock protein FRQ is a known target of temperature-responsive pathways, we asked whether VVD regulates FRQ levels or FRQ phosphorylation in a temperature-dependent manner. Both FRQ levels and phosphorylation status have been shown to undergo rhythmic changes that reflect the state of the circadian clock (Garceau et al. 1997). As described above, Neurospora liquid cultures were kept at either $21^{\circ} \mathrm{C}, 25^{\circ} \mathrm{C}$, or $28^{\circ} \mathrm{C}$ and, following a LD transfer, tissue was harvested in DD over the next $24 \mathrm{~h}$ at $4-\mathrm{h}$ intervals. Figure 5A shows two high-resolution Western blots in which several phosphorylation forms of FRQ can be distinguished. There are significant differences in the rhythm of total FRQ protein as well as in the FRQ phosphorylation pattern between wild-type and $v v d$ knockout strains. The differences in the FRQ phosphorylation pattern between strains can best be appreciated by comparison of the DD4 time points. In the vvd knockout strain at DD4, more of the lower-phosphorylated forms of FRQ are seen, whereas wild-type FRQ is hyperphosphorylated. Comparison of later time points-e.g., DD16-reveals that when levels of FRQ are low in $v v d^{\mathrm{KO}}$, they are already rising in the wild type. Consistent with the overt phenotype, total FRQ levels oscillate with an $\sim 4$-h delay in $v v d^{\mathrm{KO}}$ compared with the wild-type strain (Fig. 5B). Although the delay in the FRQ rhythm in the $v v d^{\mathrm{KO}}$ has not been shown before, it was expected because frq RNA levels display a similar 4-h shift in rhythmicity (Heintzen et al. 2001). The high resolution of FRQ phosphorylation forms allowed us to quantitate different stages of FRQ phosphorylation. For this purpose we arbitrarily assigned four groups of FRQ forms (FRQa-FRQd) spanning from the least-phosphorylated (FRQa) to the highestphosphorylated (FRQd) forms. Figure 5C shows the quantitation of the results obtained at $21^{\circ} \mathrm{C}$. In the wild type, along with increasing phosphorylation there is a dynamic shift in FRQ rhythmicity, with trough/peak levels gradually moving toward later times (phases) in DD. This result is expected, as newly synthesized FRQ is known to become phosphorylated gradually over the course of the day (Fig. 5C, left graph). However, this 


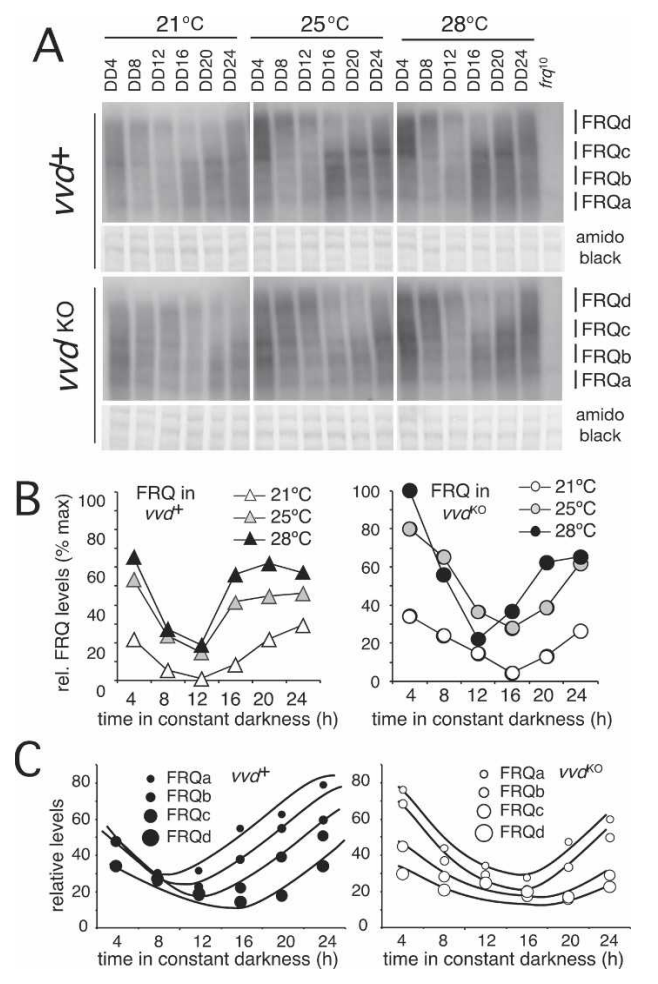

Figure 5. VVD changes the dynamics of FRQ protein phosphorylation. (A) Western blots showing FRQ levels in wild-type (top panels) and $v v d^{\mathrm{KO}}$ strains (bottom panels) at different ambient temperatures in DD. Amido black-stained membranes are shown below each blot to document even loading. (B) Quantitation of total FRQ levels using Western blots shown in $A$, graphed as the percentage of maximal FRQ expression. Peak and trough levels of total FRQ protein show a 4- to 8-h delay in ${ }_{V v} d^{\mathrm{KO}}$ (right graph) strains when compared with wild type (left graph), except at $28^{\circ} \mathrm{C}$ in a $v v d^{\mathrm{KO}}$ strain (right graph). (C) Quantitative analysis of different phosphorylation states (FRQaFRQd), as shown in $A$ at $21^{\circ} \mathrm{C}$ in DD in wild-type (left graph) and ${ }_{V V} d^{\mathrm{KO}}$ (right graph) strains graphed as the percentage of maximal expression.

change in phosphorylation is much less pronounced in a $v v d^{\mathrm{KO}}$ strain (Fig. 5C, right graph), suggesting that $v v d$ influences the dynamics of FRQ phosphorylation.

To investigate whether the differences in FRQ protein are due to a general difference in phosphorylation of total FRQ or are the result of differential accumulation of IFRQ or sFRQ, we treated total cell extracts with $\lambda$ phosphatase, a procedure that collapses phosphorylated FRQ and thus aids in the visualization of both FRQ forms (Fig. $6 \mathrm{~A})$. In line with previous reports, sFRQ does not appear to be temperature regulated in the wild type, and we show here that this is also true in the $v v d^{\mathrm{KO}}$ strain. In addition, sFRQ levels do not appear to be significantly different between strains. In Figure 6A, more 1FRQ is present in $v v d$ knockout extracts at lower temperatures compared with wild type, but lFRQ levels are not consistently higher in independent experiments. Taken together, these protein data are consistent with the $\sim 4-\mathrm{h}$ delay in overt rhythmicity of the $v v d^{\mathrm{KO}}$. We hypothesize that VVD could exert its temperature control of clock phase by regulating the timing of FRQ phosphorylation states.

\section{Discussion}

Temperature coefficients of biological phenomena vary widely. For a number of biological processes, including biological rhythms such as heart rate, the $\mathrm{Q}_{10}$ values are typically $>2$; i.e., the frequency of these rhythms at least doubles with a $10^{\circ} \mathrm{C}$ increase in temperature (Snyder 1908; Heintzen 1957). Notable exceptions are circadian rhythms, whose frequencies have $\mathrm{Q}_{10}$ values that are close to 1 , a phenomenon referred to as temperature compensation of circadian period (Pittendrigh 1954, 1993). We show here that temperature compensation of

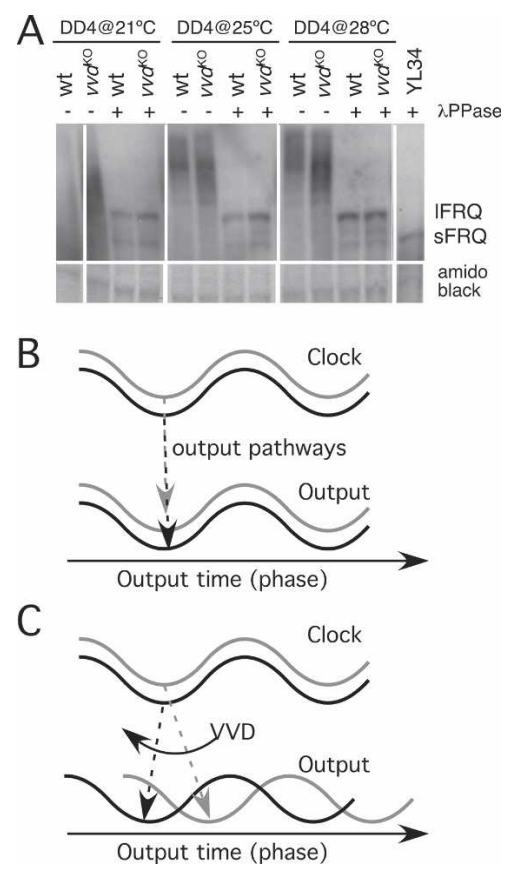

Figure 6. (A) The relative amounts of IFRQ and sFRQ are not significantly altered in $v v d^{\mathrm{KO}}$ strains, when compared with wild type (wt). Samples of total protein extracts from wild-type and $v V d^{\mathrm{KO}}$ strains harvested at DD4 at the indicated temperatures either untreated or treated with $\lambda$ phosphatase ( $\lambda$ PPase) to investigate concentrations of 1FRQ and SFRQ. The last lane shows a $\lambda$ PPase-treated protein from the strain YL34, a strain that produces only sFRQ. Amido black-stained membranes are shown below the blot to document loading. $(B, C)$ A model for the control of temperature compensation of clock-controlled output pathways (such as circadian conidiation in Neurospora). (B) A temperature-compensated clock with similar period and phase at low temperatures (gray line) or high temperatures (black line) controls temperature-independent pathways. (C) A temperature-compensated clock with similar period and phase at low temperatures (gray line) or high temperatures (black line) controlling output pathways that are temperature dependent. Increased levels of VVD at lower temperatures compensate for such temperature-dependent processes. 
the phase of clock-controlled conidiation is influenced by the PAS/LOV protein VVD. In $v v d^{\mathrm{KO}}$ mutants, the time of conidiation in the dark becomes strongly temperature dependent at the lower end of the physiologically relevant temperature range $\left(16^{\circ} \mathrm{C}-32^{\circ} \mathrm{C}\right)$, suggesting that VVD normally compensates for these temperature effects (Fig. 1). In support of this hypothesis, we found that the products of the $v v d$ gene itself are strongly temperature regulated and increase dramatically as the temperature is lowered from $25^{\circ} \mathrm{C}$ to $16^{\circ} \mathrm{C}$ (Figs. 2, 3). It appears that VVD mediates its effects on the timing of conidiation by influencing the phosphorylation kinetics of the central clock protein, FRQ (Fig. 5). Our data indicate that VVD's influence on the temperature control of the phase of conidiation is transient; i.e.,it does not permanently influence oscillator dynamics (Fig. 1; Supplementary Fig. S1). It is possible that VVD's influence on FRQ phosphorylation is also transient and may affect only a subset of FRQ phosphorylation forms that directly influence clock-controlled output pathways. This is also consistent with VVD's transient expression profile in DD.

Transient behavior of the Neurospora circadian system in the absence of VVD is reminiscent of transient phase shifts seen in other multicellular systems, such as those of plants, Drosophila, and humans after perturbation with either light or temperature pulses (Pittendrigh 1981). To explain such transients, Pittendrigh et al. (1958; Pittendrigh 1960) proposed a model in which a temperature-compensated master clock drives a temperature-dependent slave oscillator. Upon perturbation by temperature (or light), the coupling between master and temperature-sensitive slave is altered such that clock-controlled output pathways no longer reflect the time of the master clock. Only once the system has recovered from perturbation and a new steady state between master and slave is established does clock output faithfully report clock time. Not all organisms in which circadian rhythms are studied show this transient behavior (Johnson et al. 2003), and it is tempting to speculate that in these organisms mechanisms similar to the one reported here may be at work to counteract the temperature sensitivity of output pathways. Alternatively, the slave oscillator itself may be temperature compensated and act to facilitate temperature compensation of the circadian system. The regulation of VVD has properties that are consistent with such a hypothesis. VVD is strongly temperature regulated, and data presented here and in previously reported studies suggest that it is part of an autoregulatory feedback loop (Heintzen et al. 2001; Elvin et al. 2005). Slave oscillatory feedback loops have been described in other circadian systems (Heintzen et al. 1997; Staiger et al. 2003; Kornmann et al. 2007), but whether they play a role in certain aspects of temperature compensation is not known.

Temperature compensation of period is generally considered as a key factor in maintaining "target time" in entrainment conditions at different temperatures (Pittendrigh and Caldarola 1973). As far as we know, there are no reports in the literature of temperature depen- dence of circadian rhythms that are not correlated with altered clock period. Therefore, we were surprised to find that temperature compensation of period was normal in vvd deletion strains, whereas compensation of clockcontrolled phase of conidiation was not (Fig. 1B,C). Clearly, temperature compensation of phase must depend on additional temperature-controlled processes. These additional means of stabilizing clock phase may be advantageous in maintaining accurate target time in a range of ambient temperatures. This is particularly true if one considers the involvement of the clock in development, such as controlling the switch between mycelial growth and asexual spore formation in Neurospora. Although the clockwork is temperature compensated, the developmental pathways it controls may be strongly temperature dependent. In this case, without fine-tuning, appropriate phase of an output could not be preserved. For the circadian-controlled rhythm of conidiation this is indeed the case (see Supplementary Fig. S1), and the action of VVD allows appropriate timing of development.

Our results highlight the importance of temperature control of clock-regulated output pathways, an aspect of circadian rhythmicity that, surprisingly, has so far been largely ignored. It is generally believed that temperature compensation of period enables the entrained circadian system to maintain a stable phase relationship with the rhythmic environment (Pittendrigh 1993). Indeed, clock mutants with altered periods have altered phase relationships to their entrainment cycle. Similarly, entrainment of wild-type clocks to exotic LD cycles $(<24 \mathrm{~h}$ or $>24 \mathrm{~h}$ ) results in altered phase angles of entrainment (Johnson et al. 2003). However, whereas the significance of period compensation for the timing of clock-controlled processes is well supported, it neglects the fact that, at least in poikilotherms, all temperature-sensitive clock-controlled processes must also be temperature compensated. We propose a model (Fig. 6B,C) in which VVD plays a role in maintaining temperature compensation of clock output independent of temperature compensation of clock period. In this model, VVD's influence on the temperature control of the time of conidiation in the dark is in part downstream from the clock. Without VVD, the phase of conidiation is strongly temperature dependent, and the temperature-compensated clock is disengaged from one of its output targets. Our data indicate that temperature compensation of circadian period must be accompanied by temperature-compensated regulation of downstream pathways if stability of clock-controlled processes is to occur at the appropriate time of day in a range of temperatures.

\section{Materials and methods}

Strains and plasmids

All Neurospora strains in this study carry the $b d$ mutation that facilitates the assaying of clock-controlled conidiation on race tubes. General conditions for growth and manipulation are described elsewhere (Davis and deSerres 1970). Strain 87-3 (bd, a) served as laboratory clock wild type. The $v v d^{\mathrm{KO}}$ strain $(b d$, 
$v v d^{\mathrm{KO}}$ ) was generated as described previously (Heintzen et al. 2001). The $f r q^{7} ; v v d^{\mathrm{KO}}$ double mutant originated from a cross between strain 585-7 $\left(b d, f r q^{7}, a\right)$ and the laboratory strain 28910 (his-3-, bd, vvd $\left.d^{\mathrm{KO}}, A\right)$. vvd-myc and $v^{-} d^{C 108 A}$ myc strains were created by transformation of plasmids pVVDmyc1, pVVDmyc2, or pVVDCYSmyc into the laboratory strain 56-8 (his-3$\left.v v d^{\mathrm{KO}}, b d, \mathrm{~A}\right)$. In the $v v d$-myc strains, all known light and clock phenotypes of a $v v d^{\mathrm{KO}}$ strain were rescued (Supplementary Fig. S2). Therefore, in comparison with the $v v d^{C 108 A}$ myc strains, the $v v d$-myc strain is referred to in the study as a $v v d^{+}$strain.

To create the $v v d$-myc constructs, an EcoRV/EcoRI fragment containing the $v v d$ promoter, an ORF, and a corresponding 5'UTR (2401 base pairs [bp] upstream of the start codon) and 3'UTR (809 bp downstream from the stop codon) was inserted into either pDE3 $\mathrm{BH}$ (pVVDmyc1) or pBM60 (pVVDmyc2 and pVVDCYSmyc) (Margolin et al. 1997). Five myc tags amplified from pQAmyc (kindly provided by Dr. Yi Liu, Southwestern University, Dallas, TX) were inserted into the XhoI site at position 7 of the $v v d$ ORF. The $v v d^{C 108 A}$ myc construct is based on the $v v d-m y c$ construct described above, with the cysteine residue at position 108 mutated to alanine using the QuikChange site-directed mutagenesis kit (Stratagene) as described previously (Schwerdtfeger and Linden 2003). All constructs were verified by sequencing.

\section{Growth conditions}

All race tube experiments were conducted in light- and temperature-controlled chambers (Sanyo MLR-350). Race tube media contained $1 \times$ Vogel's salts, $0.1 \%$ glucose, $50 \mathrm{ng} / \mu \mathrm{L}$ biotin, $0.17 \%$ arginine, and $1.5 \%$ agar. Light intensities, whether used continuously or in light-pulse experiments, were $21 \mu \mathrm{mol} \mathrm{m} \mathrm{m}^{-2}$ $\sec ^{-1}$ (1750 lux) if not stated otherwise. Growth fronts were first marked at the LD transition and then every $24 \mathrm{~h}$ thereafter. To assay circadian period and phase in constant conditions but at different ambient temperatures, strains were grown for $1-2 \mathrm{~d}$ at either $16^{\circ} \mathrm{C}, 18^{\circ} \mathrm{C}, 21^{\circ} \mathrm{C}, 25^{\circ} \mathrm{C}, 28^{\circ} \mathrm{C}, 30^{\circ} \mathrm{C}$, or $32^{\circ} \mathrm{C}$ in $\mathrm{LL}$ and then transferred to DD at the same constant temperature. Race tube experiments shown in Supplementary Figure S1 were kept for $36 \mathrm{~h}$ in $\mathrm{LL}$ at either $18^{\circ} \mathrm{C}$ or $30^{\circ} \mathrm{C}$ before transfer to $\mathrm{DD}$ at $30^{\circ} \mathrm{C}$.

For the temperature-pulse experiments, race tube cultures were grown for $48 \mathrm{~h}$ at $25^{\circ} \mathrm{C}$ in $\mathrm{LL}$ and subsequently transferred to $25^{\circ} \mathrm{C}$ in $\mathrm{DD}$, where they were subjected to 2-h temperature pulses of either $20^{\circ} \mathrm{C}$ or $30^{\circ} \mathrm{C}$ every $2 \mathrm{~h}$ scanning the first day in DD. Phase shifts resulting from this temperature treatment were determined from at least six replicates for which the mean steady-state phase was determined using phase shifts calculated on days 2 and 3 after the temperature treatment. The center of the conidial band was used as a phase reference point and set to CT0. Circadian time $(\mathrm{CT})$ is derived by dividing the period of the rhythm into 24 equal parts. By convention, CT0 is subjective dawn and CT12 is subjective dusk. All race tubes were analyzed using CHRONO (Roenneberg and Taylor 2000).

For the light-pulse experiments, race tube cultures were grown for $48 \mathrm{~h}$ in $\mathrm{LL}$ at $28^{\circ} \mathrm{C}$ before transfer to DD at the same temperature. DD cultures were subjected to 15-min light pulses $\left(21 \mu \mathrm{mol} \mathrm{m} \mathrm{m}^{-2} \mathrm{sec}^{-1}\right)$ every $2 \mathrm{~h}$, scanning the first day in DD. Phase shifts resulting from this treatment were determined as described above for temperature PRC experiments.

\section{Liquid culture}

Mycelial mats of the strains were grown in petri dishes containing liquid culture medium ( $1 \times$ Vogel's salts, $50 \mathrm{ng} / \mu \mathrm{L}$ biotin, $0.17 \%$ arginine, $2 \%$ glucose). Mycelial disks were punched from these mats and transferred to Erlenmeyer flasks containing 50-
$100 \mathrm{~mL}$ of liquid culture medium. Flasks were shaken at 125 $\mathrm{rpm}$. In each experiment, disks were no older than $54 \mathrm{~h}$ at the time of harvest. For temperature experiments, cultures were grown at $16^{\circ} \mathrm{C}, 21^{\circ} \mathrm{C}, 25^{\circ} \mathrm{C}$, or $28^{\circ} \mathrm{C}$ for up to $36 \mathrm{~h}$ in LL before transfer to DD. Cultures were harvested at 4-h intervals over the first $24 \mathrm{~h}$ in DD. For the temperature-switch experiment, cultures were grown at $25^{\circ} \mathrm{C}$ in the light and at the light-to-dark transfer were either kept at $25^{\circ} \mathrm{C}$ or switched to $21^{\circ} \mathrm{C}$ or $28^{\circ} \mathrm{C}$. For LD entrainment experiments, cultures were grown in 12:12 $\mathrm{LD}$ conditions at a constant temperature of either $21^{\circ} \mathrm{C}$ or $28^{\circ} \mathrm{C}$.

\section{Northern blot analysis}

RNA was either extracted using the Qiagen RNeasy Mini kit according to the manufacturer's instructions for isolation of total RNA from filamentous fungus, or by previously described protocols (Elvin et al. 2005). Total RNA (7-40 $\mu \mathrm{g}$ ) was electrophoresed through a $1 \%$ agarose-formaldehyde gel, blotted onto Hybond- $\mathrm{N}^{+}$membrane (Amersham), and probed using radiolabeled antisense riboprobes (Ambion) as described previously (Elvin et al. 2005). For frq detection, nucleotides 1630-3832 (of the frq ORF) were transcribed into an antisense riboprobe using Amersham ${ }^{32} \mathrm{P}$-dUTP $(800 \mathrm{Ci} / \mathrm{mmol})$ to a specific activity of $\sim 10^{9}$ counts per minute $(\mathrm{cpm})$ per microgram. Gene-specific riboprobes for $v v d$ were obtained (AF338412, positions 239-1173) by labeling PCR fragments containing an appropriate T7 Polymerase site to generate antisense riboprobes. Membranes were hybridized in $10 \mathrm{~mL}$ of NorthernMax Prehyb/Hyb (Ambion) containing $2 \times 10^{7} \mathrm{cpm}$ per milliliter of in vitro transcribed radiolabeled probe (Ambion). Membranes were exposed to Fuji screens and were scanned using a PhosphorImager (BioRad). For signal quantification, the program Quantity One (BioRad) was used.

\section{Protein extraction and Western blots}

Protein extracts were obtained as described previously (Garceau et al. 1997). For protein phosphatase treatment, $40 \mu \mathrm{g}$ of total protein were treated with $1000 \mathrm{U}$ of $\lambda$ phosphatase according to the manufacturer's instructions (New England Biolabs). For Western blot analysis, 10-50 $\mu$ g of total protein were loaded per lane and, after SDS-PAGE, blotted onto Immobilon-P membrane (Millipore) by wet transfer. Membranes were hybridized with anti-FRQ antisera (kindly provided by Professor Jay Dunlap and Professor Jennifer Loros, Dartmouth Medical School, Hanover, NH), or monoclonal anti-MYC antibody (Santa Cruz Biotechnology) as described previously (Heintzen et al. 2001). Immunodetection was carried out using Super Signal Kit (Pierce) according to the manufacturer's instructions, and results were visualized on ECL Hyperfilm (Amersham) and the Fluor-S imager (Bio-Rad). Signals were quantitated using Quantity One (Bio-Rad).

\section{Acknowledgments}

We thank Andrew Sharrocks (University of Manchester, UK) for helpful suggestions, Yi Liu (University of Texas Southwestern Medical Center, Dallas) for kindly providing a qa-5myc construct, and Jay Dunlap and Jennifer Loros (both at Dartmouth Medical School, New Hampshire) for the generous gift of FRQ antiserum. This work was supported by a BBSRC studentship to S.H., and grants from the BBSRC to C.H. (BB/D00988X/1) and S.K.C. (BBS/B/11710).

\section{References}

Bell-Pedersen, D., Cassone, V.M., Earnest, D.J., Golden, S.S., Hardin, P.E., Thomas, T.L., and Zoran, M.J. 2005. Circadian 
rhythms from multiple oscillators: Lessons from diverse organisms. Nat. Rev. Genet. 6: 544-556.

Brunner, M. and Diernfellner, A. 2006. How temperature affects the circadian clock of Neurospora crassa. Chronobiol. Int. 23: 81-90.

Cheng, P., Yang, Y., Heintzen, C., and Liu, Y. 2001. Coiled-coil domain-mediated FRQ-FRQ interaction is essential for its circadian clock function in Neurospora. EMBO J. 20: 101108.

Cheng, P., Yang, Y.H., Gardner, K.H., and Liu, Y. 2002. PAS domain-mediated WC-1/WC-2 interaction is essential for maintaining the steady-state level of WC-1 and the function of both proteins in circadian clock and light responses of Neurospora. Mol. Cell. Biol. 22: 517-524.

Colot, H.V., Loros, J.J., and Dunlap, J.C. 2005. Temperaturemodulated alternative splicing and promoter use in the circadian clock gene frequency. Mol. Biol. Cell 16: 5563-5571.

Crosthwaite, S.K., Loros, J.J., and Dunlap, J.C. 1995. Light-induced resetting of a circadian clock is mediated by a rapid increase in frequency transcript. Cell 81: 1003-1012.

Davis, R.L. and deSerres, D. 1970. Genetic and microbial research techniques for Neurospora crassa. Methods Enzymol. 27A: 79-143.

de Paula, R.M., Lewis, Z.A., Greene, A.V., Seo, K.S., Morgan, L.W., Vitalini, M.W., Bennett, L., Gomer, R.H., and BellPedersen, D. 2006. Two circadian timing circuits in Neurospora crassa cells share components and regulate distinct rhythmic processes. J. Biol. Rhythms 21: 159-168.

Diernfellner, A.C., Schafmeier, T., Merrow, M.W., and Brunner, M. 2005. Molecular mechanism of temperature sensing by the circadian clock of Neurospora crassa. Genes \& Dev. 19: 1968-1973.

Dunlap, J.C. and Loros, J.J. 2004. The Neurospora circadian system. J. Biol. Rhythms 19: 414-424.

Dunlap, J.C., Loros, J.J., and DeCoursey, P.J. 2004. Chronobiology. Sinauer Associates, Inc., Sunderland, MA.

Elvin, M., Loros, J.J., Dunlap, J.C., and Heintzen, C. 2005. The PAS/LOV protein VIVID supports a rapidly dampened daytime oscillator that facilitates entrainment of the Neurospora circadian clock. Genes \& Dev. 19: 2593-2605.

Feldman, J.F., Pierce, S.B., and Brown, D. 1986. Temperature compensation in circadian clock mutants of Neurospora. Plant Physiol. 80 (Suppl.): 92.

Francis, C. and Sargent, M.L. 1979. Effects of temperature perturbations on circadian conidiation in Neurospora. Plant Physiol. 64: 1000-1009.

Garceau, N.Y., Liu, Y., Loros, J.J., and Dunlap, J.C. 1997. Alternative initiation of translation and time-specific phosphorylation yield multiple forms of the essential clock protein FREQUENCY. Cell 89: 469-476.

Gardner, G.F. and Feldman, J.F. 1981. Temperature compensation of circadian clock period length in clock mutants of Neurospora crassa. Plant Physiol. 68: 1244-1248.

Golden, S.S. 2003. Timekeeping in bacteria: The cyanobacterial circadian clock. Curr. Opin. Microbiol. 6: 535-540.

Gould, P.D., Locke, J.C., Larue, C., Southern, M.M., Davis, S.J., Hanano, S., Moyle, R., Milich, R., Putterill, J., Millar, A.J., et al. 2006. The molecular basis of temperature compensation in the Arabidopsis circadian clock. Plant Cell 18: $1177-1187$.

Heintzen, P. 1957. Die mathematische Behandlung der Temperaturabhängigkeit biologischer Prozesse. Pflugers Arch. 266: 214-218.

Heintzen, C. and Liu, Y. 2007. The Neurospora crassa circadian clock. Adv. Genet. 58: 25-66.

Heintzen, C., Nater, M., Apel, K., and Staiger, D. 1997. AtGRP7, a nuclear RNA-binding protein as a component of a circadian-regulated negative feedback loop in Arabidopsis thaliana. Proc. Nat1. Acad. Sci. 94: 8515-8520.

Heintzen, C., Loros, J.J., and Dunlap, J.C. 2001. The PAS protein VIVID defines a clock-associated feedback loop that represses light input, modulates gating, and regulates clock resetting. Cell 104: 453-464.

Hotta, C.T., Gardner, M.J., Hubbard, K.E., Baek, S.J., Dalchau, N., Suhita, D., Dodd, A.N., and Webb, A.A. 2007. Modulation of environmental responses of plants by circadian clocks. Plant Cell Environ. 30: 333-349.

Izumo, M., Johnson, C.H., and Yamazaki, S. 2003. Circadian gene expression in mammalian fibroblasts revealed by realtime luminescence reporting: Temperature compensation and damping. Proc. Natl. Acad. Sci. 100: 16089-16094.

Johnson, C.H., Elliott, J.A., and Foster, R. 2003. Entrainment of circadian programs. Chronobiol. Int. 20: 741-774.

Kornmann, B., Schaad, O., Bujard, H., Takahashi, J.S., and Schibler, U. 2007. System-driven and oscillator-dependent circadian transcription in mice with a conditionally active liver clock. PLOS Biol. 5: e34. doi: 10.1371/journal. pbio.0050034.

Kramer, C., Loros, J.J., Dunlap, J.C., and Crosthwaite, S.K. 2003. Role for antisense RNA in regulating circadian clock function in Neurospora crassa. Nature 421: 948-952.

Lakin-Thomas, P.L. 2006. Circadian clock genes frequency and white collar-1 are not essential for entrainment to temperature cycles in Neurospora crassa. Proc. Natl. Acad. Sci. 103: 4469-4474.

Lenz, P.H., Hower, A.E., and Hartline, D.K. 2005. Temperature compensation in the escape response of a marine copepod, Calanus finmarchius (Crustacea). Biol. Bull. 209: 75-85.

Liu, Y. and Bell-Pedersen, D. 2006. Circadian rhythms in Neurospora and other filamentous fungi. Eukaryot. Cell 5: 11841193.

Liu, Y., Garceau, N.Y., Loros, J.J., and Dunlap, J.C. 1997. Thermally regulated translational control of FRQ mediates aspects of temperature responses in the Neurospora circadian clock. Cell 89: 477-486.

Liu, Y., Merrow, M., Loros, J.J., and Dunlap, J.C. 1998. How temperature changes reset a circadian oscillator. Science 281: 825-829.

Margolin, B.S., Freitag, M., and Selker, E.U. 1997. Improved plasmids for gene targeting at the his-3 locus of Neurospora crassa by electroporation. Fung. Genet. Newsl. 44: 34-36.

Merrow, M., Brunner, M., and Roenneberg, T. 1999. Assignment of circadian function for the Neurospora clock gene frequency. Nature 399: 584-586.

Newell, R.C. 1966. The effect of temperature on the metabolism of poikilotherms. Nature 212: 427-428.

Nowrousian, M., Duffield, G.E., Loros, J.J., and Dunlap, J.C. 2003. The frequency gene is required for temperature-dependent regulation of many clock-controlled genes in Neurospora crassa. Genetics 164: 923-933.

Onai, K., Morishita, M., Itoh, S., Okamoto, K., and Ishiura, M. 2004. Circadian rhythms in the thermophilic cyanobacterium Thermosynechococcus elogantus: Compensation of the period length over a wide temperature range. J. Bacteriol. 186: 4972-4977.

Pittendrigh, C.S. 1954. On temperature independence in the clock system controlling emergence time in Drosophila. Proc. Natl. Acad. Sci. 40: 1018-1029.

Pittendrigh, C.S. 1960. Circadian rhythms and the circadian organization of living things. Cold Spring Harbor Symp. Quant. Biol. 25: 159-184.

Pittendrigh, C.S. 1981. Circadian systems: General perspective. 
Hunt et al.

In Biological rhythms (ed. J. Aschoff), pp. 57-80. Plenum, New York.

Pittendrigh, C.S. 1993. Temporal organization: Reflections of a Darwinian clock-watcher. Annu. Rev. Physiol. 55: 16-54.

Pittendrigh, C.S. and Caldarola, P.C. 1973. General homeostasis of the frequency of circadian oscillations. Proc. Natl. Acad. Sci. 70: 2697-2701.

Pittendrigh, C.S., Bruce, V., and Kaus, P. 1958. On the significance of transients in daily rhythms. Proc. Natl. Acad. Sci. 44: 965-973.

Pregueiro, A.M., Price-Lloyd, N., Bell-Pedersen, D., Heintzen, C., Loros, J.J., and Dunlap, J.C. 2005. Assignment of an essential role for the Neurospora frequency gene in circadian entrainment to temperature cycles. Proc. Natl. Acad. Sci. 102: $2210-2215$.

Rensing, L. and Ruoff, P. 2002. Temperature effect on entrainment, phase shifting, and amplitude of circadian clocks and its molecular bases. Chronobiol. Int. 19: 807-864.

Reppert, S.M. and Weaver, D.R. 2001. Molecular analysis of mammalian circadian rhythms. Annu. Rev. Physiol. 63: 647-676.

Roenneberg, T. and Taylor, W. 2000. Automated recordings of bioluminescence with special reference to the analysis of circadian rhythms. Methods Enzymol. 305: 104-119.

Roenneberg, T., Daan, S., and Merrow, M. 2003. The art of entrainment. J. Biol. Rhythms 18: 183-194.

Ruoff, P. 1992. Introducing temperature compensation in any reaction kinetic oscillator model. J. Interdiscip. Cycle Res. 23: 92-99.

Ruoff, P., Christensen, M.K., and Sharma, V.K. 2005a. PER/ TIM-mediated amplification, gene dosage effects and temperature compensation in an interlocking-feedback loop model of the Drosophila circadian clock. J. Theor. Biol. 237: 41-57.

Ruoff, P., Loros, J.J., and Dunlap, J.C. 2005b. The relationship between FRQ-protein stability and temperature compensation in the Neurospora circadian clock. Proc. Natl. Acad. Sci. 102: 17681-17686.

Schwerdtfeger, C. and Linden, H. 2001. Blue light adaptation and desensitization of light signal transduction in Neurospora crassa. Mol. Microbiol. 39: 1080-1087.

Schwerdtfeger, C. and Linden, H. 2003. VIVID is a flavoprotein and serves as a fungal blue light photoreceptor for photoadaptation. EMBO J. 22: 4846-4855.

Shrode, L.B., Lewis, Z.A., White, L.D., Bell-Pedersen, D., and Ebbole, D.J. 2001. vvd is required for light adaptation of conidiation-specific genes of Neurospora crassa, but not circadian conidiation. Fungal Genet. Biol. 32: 169-181.

Snyder, C.D. 1908. Comparative study of the temperature coefficients of the velocities of various physiological actions. Am. J. Physiol. 22: 309-329.

Somero, G.N. 1969. Enzymatic mechanisms of temperature compensation; immediate and evolutionary effects of temperature on enzymes of aquatic poikilotherms. Am. Nat. 103: $517-530$.

Somero, G.N. 2004. Adaptation of enzymes to temperature: Searching for basic 'strategies.' Comp. Biochem. Physiol. B. 139: 321-333.

Staiger, D., Zecca, L., Kirk, D.A.W., Apel, K., and Eckstein, L. 2003. The circadian clock regulated RNA-binding protein AtGRP7 autoregulates its expression by influencing alternative splicing of its own pre-mRNA. Plant J. 33: 361-371.

Tsuchiya, Y., Akashi, M., and Nishida, E. 2003. Temperature compensation and temperature resetting of circadian rhythms in mammalian cultured fibroblasts. Genes Cells 8: 713-720.
Young, M. and Kay, S.A. 2001. Timezones: A comparative genetics of circadian clocks. Nat. Rev. Genet. 2: 702-715.

Zoltowski, B.D., Schwerdtfeger, C., Widom, J., Loros, J.J., Bilwes, A.M., Dunlap, J.C., and Crane, B.R. 2007. Conformational switching in the fungal light sensor Vivid. Science 316: $1054-1057$. 


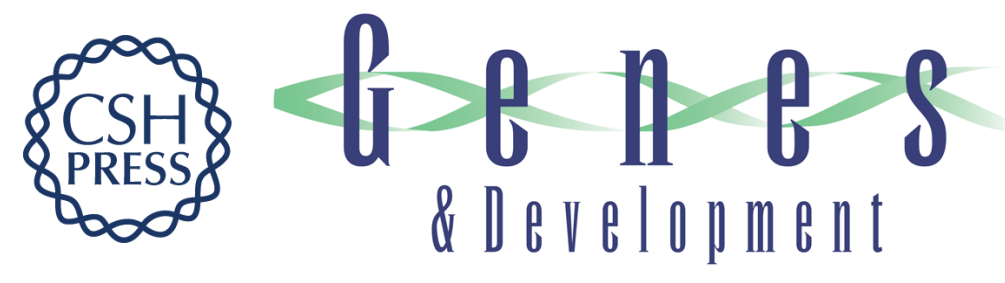

\section{The PAS/LOV protein VIVID controls temperature compensation of circadian clock phase and development in Neurospora crassa}

Suzanne M. Hunt, Mark Elvin, Susan K. Crosthwaite, et al.

Genes Dev. 2007, 21:

Access the most recent version at doi:10.1101/gad.437107

Supplemental http://genesdev.cshlp.org/content/suppl/2007/07/25/21.15.1964.DC1
Material

References This article cites 59 articles, 23 of which can be accessed free at:

http://genesdev.cshlp.org/content/21/15/1964.full.html\#ref-list-1

License

Email Alerting Receive free email alerts when new articles cite this article - sign up in the box at the top

Service

right corner of the article or click here.

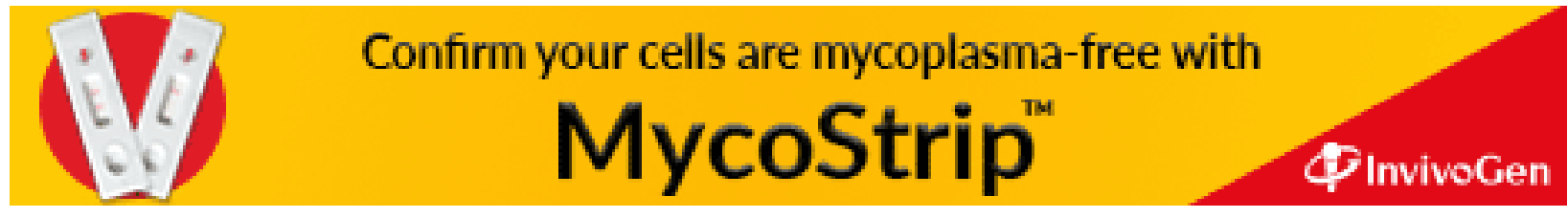

